\title{
Ganglioneuroblastoma as Vasoactive Intestinal Polypeptide-secreting Tumor: Rare Case Report in a Child
}

\author{
${ }^{1}$ Basant Kumar, ${ }^{2}$ Vijai D Upadhyaya, ${ }^{3}$ Ram Nawal Rao, ${ }^{4}$ Sheo Kumar
}

\begin{abstract}
Pathologically elevated vasoactive intestinal polypeptide (VIP) plasma levels cause secretory diarrhea with excessive loss of water and electrolyte and is characterized by the typical symptoms of hypokalemia and metabolic acidosis. It rarely occurs in patients with non-pancreatic disease. Despite the clinical severity, diagnosis of a VIP-secreting tumor is often delayed. We herein present a 14-month-old boy having prolonged therapy-resistant secretory diarrhea, persistent hypokalemia with tissue diagnosis of ganglioneuroblastoma and raised plasma VIP-levels.
\end{abstract}

Keywords: Ganglioneuroblastoma, Secretory diarrhea, VIPoma.

How to cite this article: Kumar B, Upadhyaya VD, Rao RN, Kumar S. Ganglioneuroblastoma as Vasoactive Intestinal Polypeptide-secreting Tumor: Rare Case Report in a Child. World J Endoc Surg 2015;7(2):47-50.

Source of support: Nil

Conflict of interest: None

\section{INTRODUCTION}

Firstly, Verner and Morrison (1958) described this rare syndrome of watery diarrhea, hypokalemia and achlorhydria (WDHA-syndrome) caused by hyper-secretion of vasoactive intestinal polypeptide (VIP). ${ }^{1}$ This syndrome is most commonly associated with pancreatic islet cell tumors, but rarely caused by non-pancreatic tumors, such as bronchogenic carcinoma, medullary thyroid carcinoma, retroperitoneal histiocytoma and adrenal pheochromocytoma with/without a part of multiple endocrine neoplasia (MEN) type one syndrome. ${ }^{2}$ Despite the clinical severity, diagnosis of a VIP-secreting tumor is often delayed. On literature review, we found less

\footnotetext{
${ }^{1-4}$ Associate Professor

1,2Department of Pediatric Surgery, Sanjay Gandhi Post Graduate Institute of Medical Sciences, Lucknow, Uttar Pradesh, India

${ }^{3}$ Department of Pathology, Sanjay Gandhi Post Graduate Institute of Medical Sciences, Lucknow, Uttar Pradesh India

${ }^{4}$ Department of Radiodiagnosis, Sanjay Gandhi Post Graduate Institute of Medical Sciences, Lucknow, Uttar Pradesh, India

Corresponding Author: Basant Kumar, Associate Professor Department of Pediatric Surgery, Sanjay Gandhi Post Graduate Institute of Medical Sciences, Lucknow, Uttar Pradesh, India, Phone: 05222495610, e-mail: drbasant189@gmail.com
}

than 60 cases of pediatric VIP-secreting tumors. ${ }^{3}$ Most of them are either adrenal pheochromocytoma or mixed pheochromocytoma-ganglioneuroma tumors. Mason et al, ${ }^{4}$ first described the secretory nature of neuroblastoma and vasoactive intestinal peptide (VIP) can be produced by the mature neurogenic tumors. We herein present a 14 months old boy having prolonged therapyresistant secretory diarrhea, persistent hypokalemia with tissue diagnosis of ganglioneuroblastoma and briefly review the literature.

\section{CASE REPORT}

A 14-month-old boy, weighing $9 \mathrm{~kg}$ with advanced symptoms of persistent secretory diarrhea, hypokalemia and metabolic acidosis was referred to us with radiological (computed tomography) diagnosis of retroperitoneal mass. He had growth retardation and was normotensive; extensively evaluated by the physicians. He had one previous ultrasound, reported as normal study without any comment on retroperitoneal mass. He had low grade on/off fever, abdominal pain and persistent watery diarrhea ( 25 times/day; $>1000 \mathrm{ml} /$ day; osmolar gap $<50 \mathrm{mOsm} / \mathrm{kg}$ ). Stool was brownish in color and was odorless. Abdomen was distended but soft and mass was felt on deep palpation. He accepted oral feeds but needed intravenous (I/V) fluid support along with oral and I/V potassium. Lab reports showed low hematocrit, plasma potassium and sodium levels (Hemoglobin-8 gm\%, TLC-22000 Cm, Sodium-132, Potassium-2.7, S Ferritin-32 microgm/1, S LDH-1270 IU/l). Twentyfour hour urinary catecholamines and its precursors were within normal limits but the plasma VIP level was raised $(152 \mathrm{pg} / \mathrm{ml}$ ) [normal value $<50 \mathrm{pg} / \mathrm{ml}$; done outside lab determined by radioimmunoassay]. Contrast enhanced computed tomography (CECT) showed (Figs 1A and B) a heterogeneously enhancing mass of size $5.5 \times 5.7 \times 7.1 \mathrm{~cm}$ with foci of calcification in the right anterior pararenal space, displacing almost all major vessels (celiac axis, IVC, aorta etc.), pancreas and duodenum. Paraaortic lymph nodes were enlarged with right-sided hydronephrosis. Tru-cut biopsy was performed and histopathology/immunohistochemistry revealed diagnosis of ganglioneuroblastoma (Stage-III; high-risk category; INSS). Tumor cells showed prominent nucleoli and 

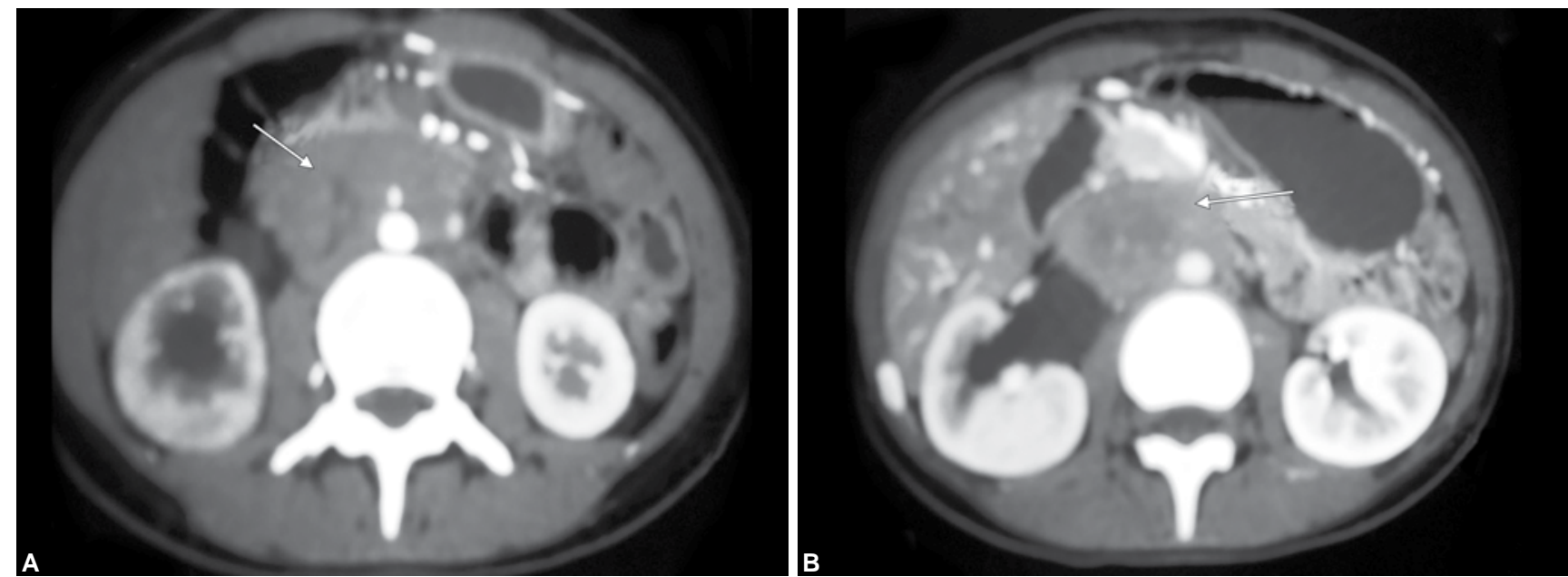

Figs 1A and B: Retroperitoneal mass displacing almost all major vessels (celiac axis, IVC, aorta etc.), pancreas and duodenum
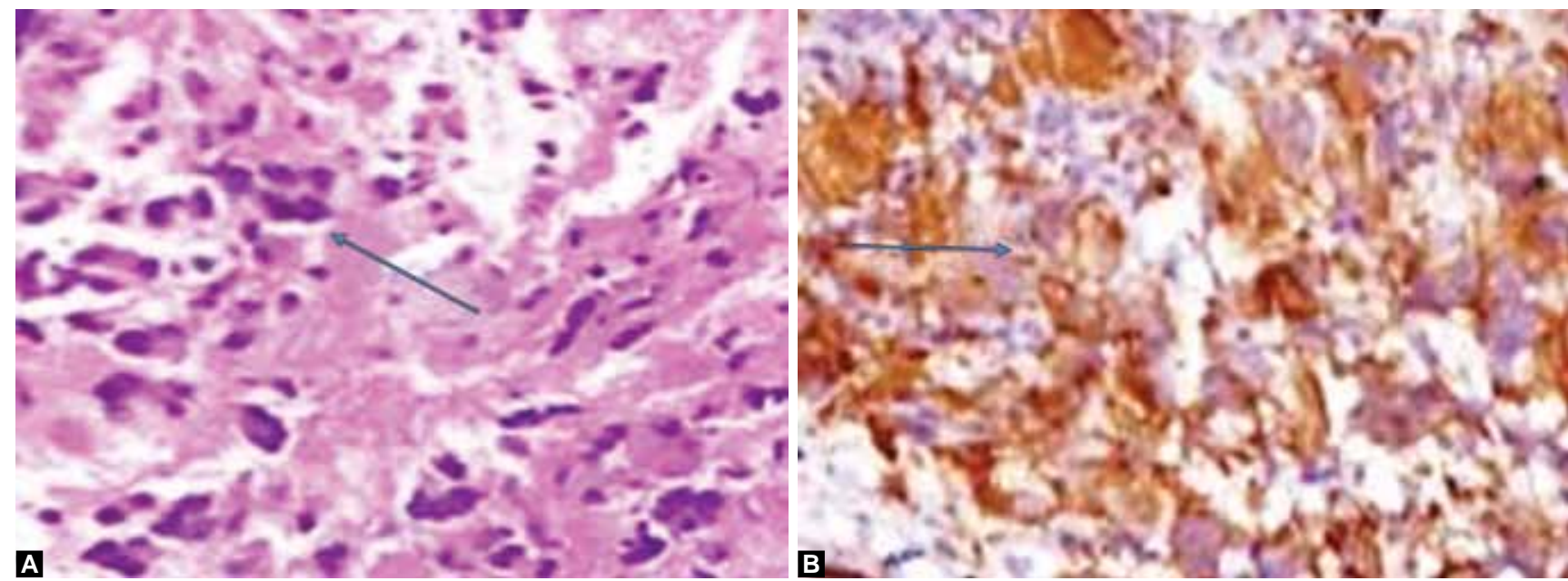

Figs 2A and B: Tumor cells showing prominent nucleoli (A) and were strongly positive for chromogranin A on immunohistochemistry (B)

were strongly positive for chromogranin A on immunohistochemistry (Figs 2A and B). Contrast enhanced computed tomography-chest, bone scan and bone marrow examination was normal without any evidence of metastasis. Child was resuscitated and optimize for neo-adjuvant chemotherapy. High-risk chemotherapy for neuroblastoma (Cyclophosphamide/Cisplatinum/Adriamycin/Etoposide) was tried and after two cycles, child showed partial response with decrease in stool frequency ( 10-14 stool/day) which was transient. We continued the chemotherapy for four cycles, but child did not show further improvement and needed continuous IV fluid and electrolyte support. In the view of patient's condition with persistent symptoms, we decided for surgery but parent of child denied for high-risk surgery and asked referral.

\section{DISCUSSION}

Neuroblastoma is a common solid tumor of childhood and arises from the neural crest cells; along the sympathetic nervous system. It is usually associated with increased production of non-epinephrine and/or its metabolites. ${ }^{4,5}$ Non-pancreatic VIP-secreting neurogenic tumors are described in literature but it is rare. Clinical experience is based mainly on case reports. Watery diarrhea, hypokalemia and achlorhydria (WDHA) syndrome is usually associated with VIP-secreting neurogenic tumors involving the retroperitoneum and mediastinum in children. Vasoactive intestinal peptide is produced mainly by ganglioneuromas, ganglioneuroblastomas, neurofibromas, pheochromocytoma or combination of pheochromocytoma-ganglioneuroma in the adrenal area. ${ }^{4,5}$ In literature, VIP production indicates a favorable prognosis. ${ }^{6}$ In contrast to individuals with VIP-secreting pancreatic tumors, patients with neurogenic lesions generally have normal serum levels of pancreatic polypeptide, gastrin, insulin, and somatostatin. ${ }^{4-6}$

A VIPoma (also known as Verner Morrison syndrome) is a rare endocrine tumor, and usually (about $90 \%$ ) arise from non- $\beta$ islet cell of the pancreas, that produce vasoactive intestinal peptide (VIP). ${ }^{1}$ Reported incidence is one per 10 million people per year. ${ }^{7}$ Vasoactive intestinal peptide, a 28-aminoacid neuropeptide, is expressed in neurons of the gastrointestinal, respiratory, and urogenital tracts and in the central and peripheral nervous 
system. It inhibits gastric acid secretion, stimulates hepatic glycogenolysis and hyperglycemia, and dilates peripheral systemic blood vessels. High plasma level causes massive secretory diarrhea, dehydration, flushing and weight loss and characteristic laboratory abnormalities include hypokalemia, achlorhydria, hypercalcemia, hyperglycemia and metabolic acidosis (WDHA-syndrome). ${ }^{1-7}$

VIPomas originate in amine precursor uptake and decarboxylation (APUD) cells of the gastroenteropancreatic endocrine system and in adrenal or extraadrenal neurogenic sites. Both chromaffin cells and ganglion cells are derived from the neural crest and have a number of common histochemical and biosynthetic characteristics. ${ }^{1,5,6}$ Sano et $\mathrm{al}^{8}$ suggested that tumor cells of hormone-producing neurogenic tumors can be divided into at least two types of cell populations. First one is adrenergic neuron-related cells containing catecholamine and somatostatin, and other one is cholinergic neuronrelated cells containing VIP. It was postulated that neoplastic growth of APUD cells may produce several polypeptides or amines, which are characteristic not only of their specific differentiated cell types, but also of associated APUD cells derived from the common neuroectoderm. ${ }^{5,8}$ VIPomas can be part of multiple endocrine neoplasia (MEN) type one syndrome. This relationship has not been observed with extra-pancreatic VIP-secreting tumors of childhood. Somatic point mutations on chromosome 11 of the MEN 1 gene have been discovered in sporadic VIPomas and VIPoma cases associated with MEN type one. ${ }^{2,5,6,8}$

VIPomas in adults are usually neuro-endocrine islet cell tumors of the pancreas while in children and adolescents; VIP is produced mainly by ganglioneuromas, ganglioneuroblastomas, neurofibromas, or other tumors in the adrenal area (the most common location) but only a small fraction of neuroblastomas and ganglioneuroblastomas produce VIP. ${ }^{9}$ Vasoactive intestinal peptide production indicates a more favorable prognosis. In contrast to individuals with VIP-secreting pancreatic tumors, patients with neurogenic lesions generally have normal serum levels of pancreatic polypeptide, gastrin, insulin, and somatostatin. ${ }^{9,10}$ VIPomas can be part of MEN 1 syndrome but this relationship has not been observed with extra-pancreatic VIP-secreting tumors of childhood. In children, it is extremely rare for a VIPoma to originate in the pancreas; instead, WDHA syndrome is usually associated with VIP-secreting neurogenic tumors involving the retroperitoneum and mediastinum. ${ }^{11}$ Pancreatic non-beta-cell hyperplasia is rare but has been reported in children. Hypokalemia and non-anion gap acidosis are the main diagnostic features of VIPomas. Hypokalemia may necessitate aggressive potassium replacement. ${ }^{9-12}$
VIPomas arise from the pancreas in $90 \%$ of cases, but they may also be found in periganglionic tissue or at other sites (e.g. colon, bronchus, adrenal glands, and liver), especially in children. ${ }^{13}$ They are almost always solitary but $<5 \%$ reported to be multicentric. Children with neuroblastomas have a relatively poor overall survival rate (30-40\%); children with VIP-secreting ganglioneuroblastomas have a considerably higher survival rate (>90\%). Approximately $50 \%$ of surgical patients with VIPoma are cured after tumor resection. In a large series of 241 adult patients with VIPomas, the 5-year survival rate was $89 \%$ among those with pancreatic VIPomas and $68.5 \%$ among those with neurogenic VIP-producing tumors. ${ }^{14}$ In metastatic disease, the 5-year survival rate was 59.6\%.

Most reports are single cases and suggest a great homogeny within this sub-group of neuroblastic tumors. The stool volume exceeds $700 \mathrm{ml}$ /day in all patients (even during fasting) and $3000 \mathrm{ml} /$ day in approximately $70 \%$. The stools are tea-colored, odorless, with features of a secretory diarrhea, such as persistence with fasting, high sodium concentration and a low stool osmolal gap in a fresh or refrigerated watery stool specimen. An osmotic gap of $>125 \mathrm{mOsm} / \mathrm{kg}$ suggests an osmotic diarrhea while a gap of $<50 \mathrm{mOsm} / \mathrm{kg}$ suggests a secretory diarrhea. Abdominal pain is mild or absent. The diagnosis of a VIP-secreting tumor is established by the presence of an otherwise unexplained high-volume secretory diarrhea and a serum VIP concentration in excess of $75 \mathrm{pg} / \mathrm{ml}$ (10-60 pg/ml). The modern treatment of neuroblastoma is determined based on risk categories. Patients are classified into low, intermediate, and high-risk categories based on the following characteristics at the time of diagnosis: stage of the disease, patient age, histologic appearance of the tumor, presence or absence of amplification of the $\mathrm{N}$-myc proto-oncogene, quantitative deoxyribonucleic acid content of the tumor (DNA index or ploidy).

Our patient had watery diarrhea from last 3 months before referral and extensively evaluated and treated (including i/v Octreotide) by pediatricians. He underwent various hematological and laboratory tests including endoscopic duodenal and colonic biopsies for definite diagnosis. Radiologist could not see the initial retroperitoneal mass on ultrasound, probably because of excessive bowel gas in dilated bowel loops due to hypokalemia. Finally, CECT-abdomen of patient showed the mass and they referred patient to us. Computed tomography-guided core biopsy from mass and its histopathological examination along with its positivity for chromogranin A on immunohistochemistry clinched the diagnosis as VIP-secreting ganglioneuroblastoma. The recommended treatment is complete surgical extirpation with or without chemotherapy. As tumor was extensive 
(high-risk category) and located near celiac axis, we tried chemotherapy to reduce the size of mass and decrease/ control the symptom of patient. Ganglioneuroblastoma is known to chemosensitive. Chemotherapy showed partial and transient effect and we decided for surgery (at least debulk the mass to control symptoms) but parents denied for high-risk surgery.

We concluded that in all patients with drug-resistant secretory diarrhea with hypokalemia and metabolic acidosis, VIP-secreting neurogenic tumors including ganglioneuroma/ganglioneuroblastoma/neuroblastoma should be considered. Diagnostic work-up must include $\mathrm{CT}$ along with other appropriate investigations.

\section{REFERENCES}

1. Verner JV, Morrison AB. Islet cell tumor and a syndrome of refractory waterydiarrheaandhypokalemia.AmJMed 1958Sep; 25(3):374-380.

2. Said SI. Evidence for secretion of vasoactive intestinal peptide by tumors of pancreas, adrenal medulla, thyroid and lung: support for the unifying APUD concept. Clin Endocrinol (Oxf) 1976;Suppl 5:201S-204S.

3. Reindl T, Degenhardt P, Luck W, Riebel T, Sarioglu N, et al. The VIP-secreting tumor as a differential diagnosis of protracted diarrhea in pediatrics. Klin Padiatr 2004;216(5):264-269.

4. Mason GA. Hart-Mercer J, Millar EJ, Strang LB, Wynne NA. Adrenaline secreting neuroblastoma in an infant. Lancet 1957;273(6990):322-325.
5. Ikuta SI, Yasui C, Kawanaka M, Aihara T, Yoshie H, et al. Watery diarrhea, hypokalemia and achlorhydria syndrome due to an adrenal pheochromocytoma. World J Gastroenterol 2007;13(34):4649-4652.

6. El Shafie M, Samuel D, Klippel CH, Robinson MG, Cullen BJ. Intractable diarrhea in children with VIP-secreting ganglioneuroblastomas. J Pediatr Surg 1983;18(1):34-36.

7. Friesen SR. Update on the diagnosis and treatment of rare neuroendocrine tumors. Surg Clin North Am 1987;67(2): 379-393.

8. Sano T, Saito H, Inaba H, Hizawa K, Saito S, et al. Immunoreactive somatostatin and vasoactive intestinal polypeptide in adrenal pheochromocytoma. An immuno-chemical and ultrastructural study. Cancer 1983;52(2):282-289.

9. Batcher E, Madaj P, Gianoukakis AG. Pancreatic neuroendocrine tumors. Endocr Res 2011;36(1):35-43.

10. Rescorla FJ, Vane DW, Fitzgerald JF, West KW, Grosfeld JL. Vasoactive intestinal polypeptide-secreting ganglioneuromatosis affecting the entire colon and rectum. J Pediatr Surg 1988;23(7):635-637.

11. Grier JF. WDHA (watery diarrhea, hypokalemia, achlorhydria) syndrome: clinical features, diagnosis, and treatment. South Med J 1995;88(1):22-24.

12. Quak SH, Prabhakaran K, Kwok R, O'Reilly AP. Vasoactive intestinal peptide secreting tumors in children: a case report with literature review. Aust Pediatr J 1988;24(1):55-58.

13. Doherty GM. Rare endocrine tumors of the GI tract. Best Pract Res Clin Gastroenterol 2005;19(5):807-817.

14. Müller S, Kupka S, Königsrainer I, Northoff H, Sotlar K, Bock T. MSH2 and CXCR4 involvement in malignant VIPoma. World J Surg Oncol 2012;10:264. 